\title{
Demonstration of a diode-pumped metastable Ar laser
}

\author{
Jiande Han, ${ }^{1}$ Leonid Glebov, ${ }^{2}$ George Venus, ${ }^{2}$ and Michael C. Heaven ${ }^{1, *}$ \\ ${ }^{1}$ Department of Chemistry, Emory University, Atlanta, Georgia 30322, USA \\ ${ }^{2}$ The College of Optics \& Photonics, University of Central Florida, Orlando, Florida 32816, USA \\ ${ }^{*}$ Corresponding author: mheaven@emory.edu
}

Received September 30, 2013; revised November 11, 2013; accepted November 11, 2013; posted November 12, 2013 (Doc. ID 198306); published December 13, 2013

\begin{abstract}
Pulsed lasing from optically pumped rare gas metastable atoms ( $\mathrm{Ne}, \mathrm{Ar}, \mathrm{Kr}$, and $\mathrm{Xe}$ ) has been demonstrated previously. The laser relies on a three-level scheme, which involves the $(n+1) p[5 / 2]_{3}$ and $(n+1) p[1 / 2]_{1}$ states from the $n p^{5}(n+1) p$ electronic configuration and the metastable $(n+1) s[3 / 2]_{2}$ level of the $n p^{5}(n+1) s$ configuration (Racah notation). Population inversions were achieved using relaxation from $\left((n+1) p[5 / 2]_{3}\right.$ to $(n+1) p[1 / 2]_{1}$ induced by collisions with helium or argon at pressures near $1 \mathrm{~atm}$. Pulsed lasing was easily achieved using the high instantaneous pump intensities provided by a pulsed optical parametric oscillator excitation laser. In the present study we examine the potential for the development of a continuous wave (CW) optically pumped Ar laser. We report lasing of the $4 p[1 / 2]_{1} \rightarrow 4 s[3 / 2]_{2}(912.547 \mathrm{~nm})$ transition following CW diode laser excitation of the $4 p[5 / 2]_{3} \leftarrow 4 s[3 / 2]_{2}$ line $(811.754 \mathrm{~nm})$. A pulsed discharge was used to generate $\operatorname{Ar} 4 s[3 / 2]_{2}$, and the time-resolved lasing kinetics provide insights concerning the radiative and collisional relaxation processes. (c) 2013 Optical Society of America

OCIS codes: (140.1340) Atomic gas lasers; (140.3380) Laser materials; (140.3460) Lasers; (140.3480) Lasers, diodepumped.

http://dx.doi.org/10.1364/OL.38.005458
\end{abstract}

Diode-pumped alkali vapor lasers (DPALs) have been actively developed in recent years [1-7] due to their many attractive features. These include good beam quality, high quantum efficiencies, lasing in the near-IR region, and the potential for scaling to high powers. DPALs exploit the strongly allowed $n p \leftarrow n s$ transitions of the alkali atoms, where $n=4,5$, and 6 for $\mathrm{K}, \mathrm{Rb}$, and $\mathrm{Cs}$, respectively. Lasing is achieved using a standard threeenergy-level scheme. The $D_{2}$ transition $\left({ }^{2} \mathrm{P}_{3 / 2} \leftarrow{ }^{2} \mathrm{~S}_{1 / 2}\right)$ is used for optical excitation, and the atoms lase on the $D_{1}$ line $\left({ }^{2} \mathrm{P}_{1 / 2} \rightarrow{ }^{2} \mathrm{~S}_{1 / 2}\right)$. Population inversions are realized by rapid collision-induced spin-orbit energy transfer between the ${ }^{2} \mathrm{P}_{3 / 2}$ and ${ }^{2} \mathrm{P}_{1 / 2}$ levels. It has been found that saturated hydrocarbons, such as methane and ethane, are effective energy transfer agents that do not quench the ${ }^{2} \mathrm{P}_{J}$ states (in this context quenching denotes nonradiative relaxation to the ${ }^{2} \mathrm{~S}_{1 / 2}$ ground state). Proof-ofprinciple experiments have demonstrated high optical conversion efficiencies. However, there are chemistryrelated challenges. Alkali metal vapors are highly reactive and somewhat difficult to handle. The optically excited atoms slowly react with the hydrocarbons, leading to consumption of the alkali metal, precipitation of particulates, and contamination of the gain cell optics. Efforts to identify nonreactive energy transfer agents are in progress. Helium can be used, but undesirably high pressures are required [ $[\underline{9}, \underline{9}]$. The heavier rare gases $(\mathrm{Rg})$ are ineffective as collisional transfer agents, but DPAL systems that rely on direct optical excitation of collision pairs have been reported for $\mathrm{Rg}=\mathrm{Ar}, \mathrm{Kr}$, and $\mathrm{Xe}$ [10-15]. The challenge for collision-pair excitation is that very high pump powers are needed to reach threshold gain.

As an alternative, we have considered options for optically pumped atomic lasers that utilize inert gas mixtures [16]. The $\mathrm{Rg}$ atoms have metastable $n p^{5}(n+1) s$ $[3 / 2]_{2}$ states that are readily generated using gas discharges. The $(n+1) p \leftarrow(n+1) s$ transitions of these metastables are entirely analogous to the $n p \leftarrow n s$ transitions of the alkali atoms [17], the primary difference being the more complicated electronic energy-level structure arising from the presence of an open shell $n p^{5}$ ion core. A three-level laser scheme for the metastable $\mathrm{Rg}$ atoms can be constructed using excitation from the $(n+1) s[3 / 2]_{2}$ state, as shown for Ar in Fig. 1 .

The laser is excited using the $(n+1) p[\overline{5} / 2]_{3} \leftarrow$ $(n+1) s[3 / 2]_{2}$ transition and lases, following collisional relaxation, on the $(n+1) p[1 / 2]_{1} \rightarrow(n+1) s[3 / 2]_{2}$ line. The $(n+1) p[5 / 2]_{3} \rightarrow(n+1) p[1 / 2]_{1}$ energy transfer step is accomplished using $\mathrm{He}$ and/or the lasing Rg. For this process to be effective, the device needs to operate at pressures in excess of 0.5 bar.

Previously, we used pulsed excitation techniques to demonstrate the feasibility of this approach [16]. Metastable states of $\mathrm{Ar}, \mathrm{Kr}$, and Xe were populated using a pulsed discharge. Typically, gas mixtures consisting of about 0.04 mbar of the heavier Rg in $0.5-2.0$ bar of He were employed. Optical excitation was provided by a tunable optical parametric oscillator system. This laser provided pulse energies near $0.25 \mathrm{~mJ}$ with a pulse duration of 10 ns. For the beam diameter used, the peak excitation fluence was close to $0.2 \mathrm{MW} \mathrm{cm}^{-2}$. Lasing was readily obtained for $\mathrm{Ar}, \mathrm{Kr}$, and Xe. For Ar it was also found that lasing could be achieved using the pure gas at a total pressure of 1 bar. More recently, we have demonstrated a pulsed, optically pumped Ne laser.

In principle, continuous wave (CW) operation of the optically pumped Rg lasers is possible, and there are directed energy applications that require $\mathrm{CW}$ or longpulse output. As for DPAL systems, it is desirable to be able to pump the Rg lasers using commercially available diode-laser sources. As a step toward the development of a $\mathrm{CW}$ device, we have investigated the question of whether the optical power available from a small diode laser (in the $5-10 \mathrm{~W}$ range) would be sufficient to invert the $4 p[1 / 2]_{1} \rightarrow 4 s[3 / 2]_{2}$ transition of Ar.

Figure 2 shows the experimental apparatus used for $\mathrm{Ar}$ lasing experiments with a CW diode pump laser. The metastable $4 s[3 / 2]_{2}$ atoms were generated by an electrical discharge in a mixture of Ar and He. As noted above, the total gas pressure needed to be above 0.5 bar in order 


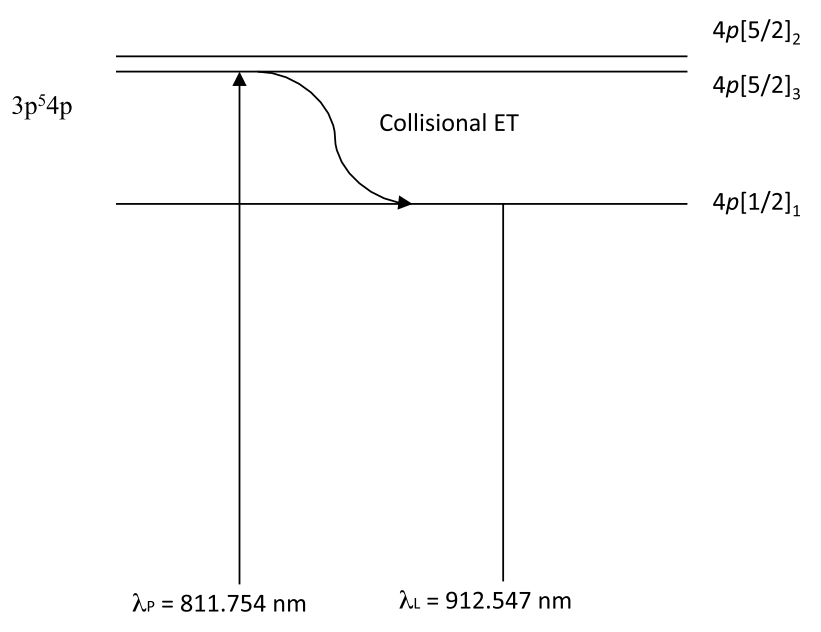

was used to evacuate the cell to a base pressure of 0.02 mbar.

Ar and He gases were flowed through the cell, and the mixture composition was controlled by metering valves on each of the gas inlet lines. The total cell pressure was controlled by means of a throttle valve in the line to the vacuum pump. Flow rates were monitored by digital flow meters (Omega FMA 1820 and 1812), and the total pressure was measured by a capacitance manometer (MKS Baratron). Liquid nitrogen cooled traps were used on both the inlet and outlet sides of the discharge cell in order to further purify the reagent gases and to prevent oil vapor back-streaming from the mechanical pump. The ultrahigh-purity grade of argon and helium was used for these experiments.

For the discharge, -1000 to $-2000 \mathrm{~V}$ pulses of about $1 \mu$ s duration were generated using a high-voltage power supply (SRS, PS350/5000V-25W) and a high-voltage pulse generator (DEI, PVX4140). The latter was triggered by a time delay generator (SRS, DG535). Stable operation at pressures around 0.5 bar was achieved using a pulse repetition frequency of $1 \mathrm{kHz}$. With freshly cleaned electrodes, stable glow discharges could be obtained for pressures up to 130 mbar of pure argon and 460 mbar for mixtures containing a small amount of argon in helium. The CW optical pump source was constructed using a high-power laser diode in an external resonator $[18,19]$. It was built around a single diode (stripe width of $1 \mathrm{~mm}$ ) that lased at wavelengths close to $811 \mathrm{~nm}$ (Axcel Phononics). The lasing wavelength was locked by an external volume Bragg grating (VBG) produced at OptiGrate. The emission spectrum width was narrower than $10 \mathrm{GHz}$. The spectral locking was stable up to the full pumping current. Thermal control of the VBG enabled fine tuning of the emission wavelength over a range of $0.4 \mathrm{~nm}$. At the

Fig. 1. Partial energy-level diagram for the states of Ar derived from the $3 p^{5} 4 s$ and $3 p^{5} 4 p$ electronic configurations. Transitions between the three levels for an optically pumped Ar laser are shown.

to sustain sufficiently high collisional energy-transfer rates. We did not have access to a power supply that was capable of maintaining a DC or RF discharge under these high-pressure conditions. Consequently, a pulsed DC discharge was used for these tests. The discharge was struck between a pair of parallel stainless steel plates $(2.5 \mathrm{~cm} \times 2.5 \mathrm{~cm})$ that were separated by $0.5 \mathrm{~cm}$. These electrodes were mounted in a cylindrical glass cell that was $15 \mathrm{~cm}$ long with a diameter of $5 \mathrm{~cm}$. The ends were closed by flat glass windows. A mechanical pump

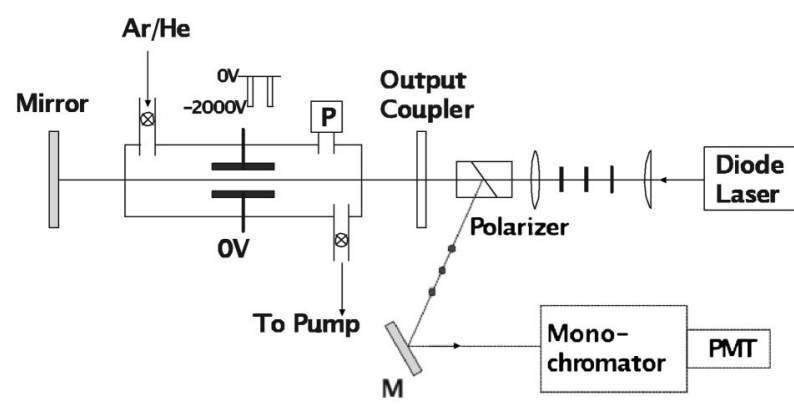

Fig. 2. Experimental setup for demonstrating Ar lasing with a CW diode pump source. $\operatorname{Ar} 4 p[5 / 2]_{3} \leftarrow 4 s[3 / 2]_{2}$ pumping wavelength (811.754 $\mathrm{nm}$ ), the diode laser provided a maximum power of about $8 \mathrm{~W}$ with a driving current of $11 \mathrm{~A}$. The fast axis of the emitted beam was collimated with divergence close to the diffraction limit. The slow axis was not collimated, having divergence of approximately $8^{\circ}$. A cylindrical lens was used to collimate along this axis. The pump laser beam was then focused at the center of the discharge electrodes by a $20 \mathrm{~cm}$ focal length plano-convex lens. At the focus, the diameter of the beam waist was approximately $200 \mu \mathrm{m}$. A plane parallel cavity was formed from a total reflector and an output coupler that consisted of an uncoated glass flat. This choice was determined by the optics available in our facility. The optical cavity length was $35 \mathrm{~cm}$. The reflectivity of the glass flat was approximately $8 \%$.

A Glan-Tylor polarizer was used to separate the lasing from the pump radiation. The pump beam was horizontally polarized, and the vertically polarized component of the lasing was directed to a monochromator. Lasing at $912.547 \mathrm{~nm}$ was detected by a photomultiplier and recorded using a digital oscilloscope (LeCroy, WaveSurfer 24Xs).

With pulsed discharge generation of $\mathrm{Ar} 4 s[3 / 2]_{2}$ and diode laser excitation of the $4 p[5 / 2]_{3} \leftarrow 4 s[3 / 2]_{2}$ transition, transient lasing was observed from $4 p[1 / 2]_{1} \rightarrow 4 s[3 / 2]_{2}$. The lasing power was sensitive to the alignment of the cavity. Figure 3 shows two lasing time profiles, recorded using the digital oscilloscope. These experiments were 


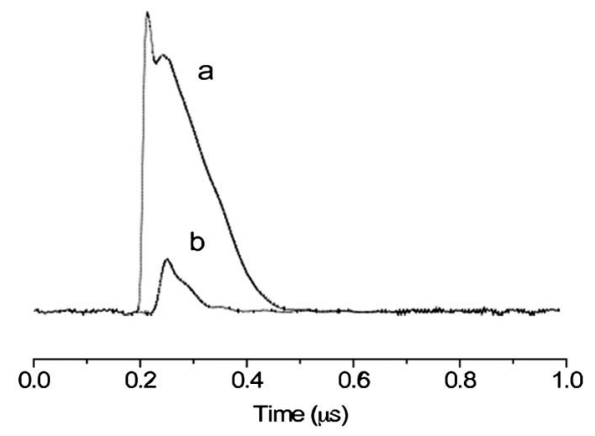

Fig. 3. Transient lasing time profiles from $\operatorname{Ar} 4 p[1 / 2]_{1} \rightarrow$ $4 s[3 / 2]_{2}$. (a) Output coupler aligned. (b) Output coupler misaligned.

carried out with 39.5 mbar of Ar in 421 mbar of helium. The taller trace in Fig. 3 corresponds to a well-aligned output coupler, while the weaker trace was recorded with the output coupler misaligned. The nonlinear dependence of the signal on the cavity alignment confirms that the system is lasing and not simply emitting laser-induced fluorescence. Note that the pump laser was free running throughout these measurements.

Figure 4 shows a plot of the output power versus pump laser power. The lasing threshold was slightly above $3 \mathrm{~W}$, and the output power was almost linearly dependent on the pump power above $4 \mathrm{~W}$. The absolute output power was not determined as a suitable power meter was not available.

Lasing of pure Ar was achieved previously using pulsed optical excitation [16], so we investigated the possibility that pure Ar could also lase with CW diode pumping. For these experiments it proved to be difficult to maintain a stable glow discharge in Ar, even at pressures as low as 130 mbar. At pressures above 120 mbar, the discharge was liable to generate filaments and arcing. Despite being limited to a lower pressure range by the discharge instabilities, optically pumped lasing was observed for pure Ar at a pressure of 90 mbar.

The electrical discharge is a complex environment where the $4 s[3 / 2]_{2}$ metastable density and the excited state relaxation kinetics are determined by many factors.

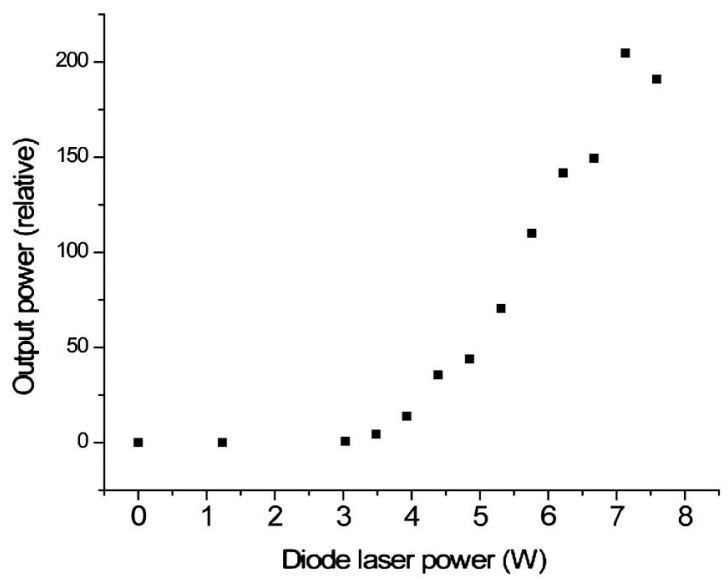

Fig. 4. Lasing power verses diode pump power. Gas mixture: Ar, 33 mbar; He, 423 mbar.
These include the composition of the gas mixture, the geometry of the electrodes, the discharge voltage (and current), and the temporal profile of the discharge pulse. In this preliminary study, we briefly examined the influence that some of these factors have on the laser output power. The dependence on the gas composition was examined by first changing the Ar:He ratio at a fixed total pressure of 395 mbar. The power increased rapidly over the Ar pressure range 20-30 mbar, but the rate of change decreased at higher partial pressures, flattening out over the 40-66 mbar range. Above this partial pressure, the $\mathrm{Ar} / \mathrm{He} 395$ mbar discharge became unstable. The effect of the He pressure was examined for a constant flow of $\mathrm{Ar}$ (corresponding to a partial pressure of approximately 30 mbar). The lasing power increased with increasing He pressure over the range 130-460 mbar. Attempts to go to higher pressures were unsuccessful due to discharge instabilities.

The dependence of the lasing temporal shape on the discharge voltage was examined using a mixture of 40 mbar of Ar in 420 mbar of He. A voltage of $-1100 \mathrm{~V}$ was sufficient to produce a glow discharge, but lasing was not observed for voltages below $-1200 \mathrm{~V}$. As can be seen in Fig. 5 , both the peak intensity and the duration of the laser pulse increased with increasing voltage over the range -1200 to $-2000 \mathrm{~V}$. Beyond this range the discharge became unstable and the power decreased.

Time-resolved detection of the emission from the discharge indicated that the Ar metastables were primarily produced during the initial gas breakdown. Without optical excitation, the Ar metastables generated by the DC discharge were expected to survive for several microseconds after they had been produced. Two measurements were made to verify this assumption. In the first test, a fraction of the diode laser beam was used to monitor the metastables via the $4 p[5 / 2]_{3} \leftarrow 4 s[3 / 2]_{2}$ absorption line. The transient absorption signal decayed to half of the initial value in approximately $7 \mu \mathrm{s}$, with the first $4 \mu \mathrm{s}$ showing a much slower decay. For the second test, a delayed pulsed dye laser was used to excite fluorescence using the $4 p[3 / 2]_{2}-4 s[3 / 2]_{2}$ transition. The temporal profile for the $4 s[3 / 2]_{2}$ state population, recovered by

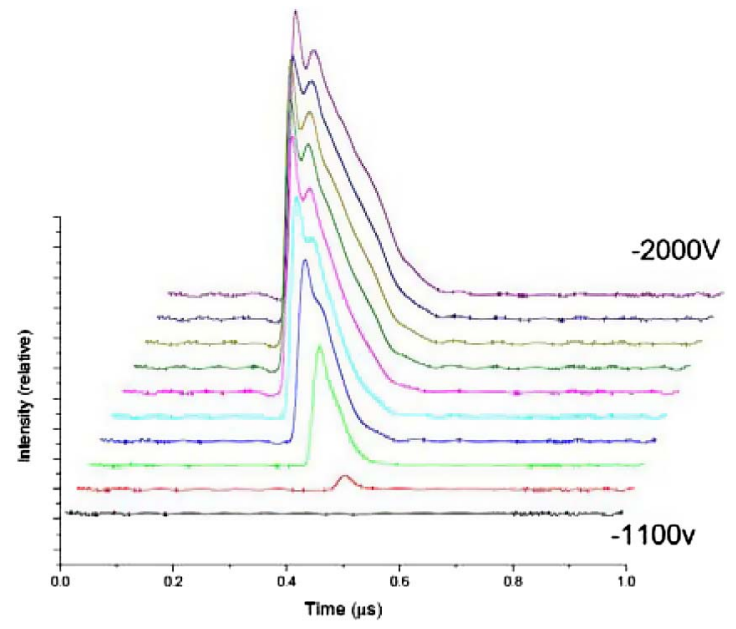

Fig. 5. Dependence of the lasing temporal shape on the discharge voltage. 
sweeping the delay between the discharge and probe laser pulses, yielded essentially the same result as the transient absorption measurement.

Given this persistence of the Ar metastables, it was surprising to find that the laser pulses induced by $\mathrm{CW}$ pumping were of relatively short duration (50-200 ns, cf. Figs. 3 and 5 ). This behavior suggests that the metastables were being removed by processes associated with the optical pumping. To check for collisional removal of the $4 p[5 / 2]_{3}$ and $4 p[1 / 2]_{1}$ state populations by processes occurring in the discharge, we examined the fluorescence decay kinetics of these levels using pulsed laser excitation. Fluorescence decay curves recorded during and after the discharge were the same, within the experimental errors. Hence, deactivation of the upper levels of the laser system did not appear to be the cause of the optically induced loss of metastables.

Preliminary modeling indicates that radiative transfer to the $4 s[3 / 2]_{1}$ level is the cause of the loss of population from $4 s[3 / 2]_{2}$. The pump transition does not contribute to this transfer directly. Due to the high electronic angular momentum of $4 p[5 / 2]_{3}$, this state can only radiate back to $4 s[3 / 2]_{2}$. However, the upper laser level can radiate back to all four of the $3 p^{5} 4 s$ states. The dominant transition is the laser line, which has an Einstein spontaneous emission coefficient of $1.89 \times 10^{7} \mathrm{~s}^{-1}$. Transitions down to the $4 s[3 / 2]_{1}, 4 s[1 / 2]_{0}$, and $4 s[1 / 2]_{1}$ states account for $26 \%$ of the total spontaneous decay rate, with Einstein coefficients of $5.43 \times 10^{6}, 9.8 \times 10^{5}$, and $1.9 \times 10^{5} \mathrm{~s}^{-1}$, respectively [20]. If the channels that repopulate $4 s[3 / 2]_{2}$ are not sufficiently rapid, transfer via the $4 s[3 / 2]_{2} \rightarrow$ $4 p[5 / 2]_{3} \rightarrow 4 p[1 / 2]_{1} \rightarrow 4 s[3 / 2]_{1}$ sequence terminates the laser. We have noted that the discharge only produces significant quantities of Ar metastables during the initial gas breakdown. The short duration of the laser pulse implies that He-induced collisional energy transfer from $4 s[3 / 2]_{1}$ to $4 s[3 / 2]_{2}$ is slow. Two-laser pump probe experiments indicate that the transfer rate is about $10^{6} \mathrm{~s}^{-1}$ for a He partial pressure of 400 mbar [21].

The present study shows that a metastable Ar laser can be optically pumped using intensities that are comfortably within the range of commercially available diode laser sources. The lasing threshold was observed near $11 \mathrm{~kW} \mathrm{~cm}^{-2}$, far below the peak power used in the earlier pulsed laser excited Ar laser [16]. The lasing and discharge kinetics observed here highlight a key issue for the further development of this laser concept. It will be necessary to identify a discharge configuration that is capable of continuous production of the $(n+1) s[3 / 2]_{2}$ metastables. Ideally, the discharge will operate at pressures of $1 \mathrm{bar}$ or greater, to facilitate energy transfer between the $(n+1) s[3 / 2]_{J}$ states.
We thank Dr. Gordon Hager for helpful discussions of the optical pumping kinetics and Vadim Smirnov for VBG recording. This work was supported by the Joint Technology Office through the Air Force Office of Scientific Research (AFOSR) under contracts FA9550-07-1-0572 and FA9550-13-1-0002.

\section{References}

1. B. D. Barmashenko, S. Rosenwaks, and M. C. Heaven, Opt. Commun. 292, 123 (2013).

2. R. J. Beach, W. F. Krupke, V. K. Kanz, S. A. Payne, M. A. Dubinskii, and L. D. Merkle, J. Opt. Soc. Am. B 21, 2151 (2004).

3. A. V. Bogachev, S. G. Garanin, A. M. Dudov, V. A. Yeroshenko, S. M. Kulikov, G. T. Mikaelian, V. A. Panarin, V. O. Pautov, A. V. Rus, and S. A. Sukharev, Quantum Electron. 42, 95 (2012).

4. K. L. Galbally-Kinney, D. L. Maser, W. J. Kessler, W. T. Rawlins, and S. J. Davis, Appl. Phys. Lett. 100, 041110 (2012).

5. N. D. Zameroski, G. D. Hager, W. Rudolph, and D. A. Hostutler, J. Opt. Soc. Am. B 28, 1088 (2011).

6. B. Zhdanov, J. Sell, and R. J. Knize, Electron. Lett. 44, 582 (2008).

7. C. V. Sulham, G. P. Perram, M. P. Wilkinson, and D. A. Hostutler, Opt. Commun. 283, 4328 (2010).

8. S. S. Q. Wu, T. F. Soules, R. H. Page, S. C. Mitchell, V. K. Kanz, and R. J. Beach, Opt. Lett. 32, 2423 (2007).

9. S. S. Q. Wu, T. F. Soules, R. H. Page, S. C. Mitchell, V. K. Kanz, and R. J. Beach, Opt. Commun. 281, 1222 (2008).

10. J. D. Readle, C. J. Wagner, J. T. Verdeyen, T. M. Spinka, D. L. Carroll, and J. G. Eden, Appl. Phys. Lett. 94, 251112 (2009).

11. J. D. Readle, C. J. Wagner, J. T. Verdeyen, D. A. Carroll, and J. G. Eden, Electron. Lett. 44, 1466 (2008).

12. J. D. Readle, J. T. Verdeyen, J. G. Eden, S. J. Davis, K. L. Gabally-Kinney, W. T. Rawlins, and W. J. Kessler, Opt. Lett. 34, 3638 (2009).

13. J. D. Hewitt and J. G. Eden, Appl. Phys. Lett. 101, 241109 (2012).

14. J. D. Hewitt, T. J. Houlahan, Jr., J. E. Gallagher, D. L. Carroll, A. D. Palla, J. T. Verdeyen, G. P. Perram, and J. G. Eden, Appl. Phys. Lett. 102, 111104 (2013).

15. J. D. Hewitt, J. D. Readle, and J. G. Eden, Appl. Phys. Lett. 101, 071102 (2012).

16. J. Han and M. C. Heaven, Opt. Lett. 37, 2157 (2012).

17. D. W. Setser, T. D. Dreiling, H. C. Brashears, Jr., and J. H. Kolts, Faraday Discuss. Chem. Soc. 67, 255 (1979).

18. A. Gourevitch, G. Venus, V. Smirnov, and L. Glebov, Opt. Lett. 32, 2611 (2007).

19. A. Gourevitch, G. Venus, V. Smirnov, D. A. Hostutler, and L. Glebov, Opt. Lett. 33, 702 (2008).

20. NIST, "NIST atomic spectra database," http://www.nist.gov/ $\mathrm{pml} /$ data/asd.cfm (2013).

21. J. Han and M. C. Heaven are preparing a manuscript to be called "Relaxation of the $\operatorname{Ar} 3 p^{5} 4 s$ states induced by collisions with He." 